\title{
El lado oscuro de Internet: un estudio bibliométrico de la literatura sobre comportamientos adictivos en línea
}

\author{
Rosa Lidia Vega-Almeida*, Ricardo Arencibia-Jorge** \\ * Centro de Histoterapia Placentaria (CHP-BioCubaFarma), La Habana, Cuba. \\ Correo-e: vega.rosa@infomed.sld.cu | ORCID iD: https://orcid.org/0000-0003-4203-6207 \\ ** Empresa de Tecnologías de la Información (ETI-BioCubaFarma), La Habana, Cuba. \\ Correo-e: ricardo.arencibia@eti.biocubafarma.cu | ORCID iD: https://orcid.org/0000-0001-8907-2454
}

Recibido: 24-09-2018; 2a versión: 03-05-2019; Aceptado: 08-05-2019.

Cómo citar este artículo/Citation: Vega-Almeida, R. L.; Arencibia-Jorge, R. (2019). El lado oscuro de Internet: un estudio bibliométrico de la literatura sobre comportamientos adictivos en línea. Revista Española de Documentación Científica, 42 (4), e251. https://doi.org/10.3989/redc.2019.4.S8

Resumen: El presente estudio bibliométrico caracterizó la investigación sobre problemas médicos derivados del uso excesivo o inadecuado de Internet durante el período 2011-2017. Se identificaron en PubMed 1.178 artículos sobre conductas adictivas, que constituyeron el 2,7\% de la literatura biomédica mundial sobre Internet, y el 22,3\% de los trabajos que tienen a Internet como tópico principal. Se analizó el comportamiento anual de la producción científica, se identificaron las revistas y autores más productivos, y se determinaron los principales frentes de investigación a partir de técnicas bibliométricas. La adicción a los juegos en línea y la ciberpornografía, las alteraciones cognitivas asociadas a la adicción a Internet, y el sobreuso de teléfonos inteligentes y redes sociales, constituyen crecientes problemáticas identificadas en la literatura. Es indispensable el desarrollo de políticas públicas que estimulen el aprovechamiento de las ventajas de Internet y minimicen los riesgos de conductas patológicas en niños y adolescentes.

Palabras clave: adicción a internet; uso patológico de Internet; producción científica; estudio bibliométrico; visualización de frentes de investigación.

\section{The dark side of Internet: a bibliometric study of online addictive behaviors}

Abstract: The current bibliometric study characterized the research on medical problems derived from the excessive or inappropriate use of Internet during the period 2011-2017. A total of 1.178 papers on addictive behaviors were identified in PubMed, which accounts for $2.7 \%$ of Internet-related biomedical literature, and $22.3 \%$ of papers having Internet as the main topic. The annual behavior of scientific output was analyzed, the most productive journals and authors were identified, and the main research fronts were recognized through bibliometric techniques. Addictions to online gambling and cyberpornography, cognitive alterations associated to Internet addiction and overuse of smartphones and social networks were the main problems identified in the scientific literature. As a conclusion, is essential the development of public policies to take advantage of the Internet benefits, minimizing the risks of pathological behavior in children and adolescents.

Keywords: internet addiction; pathological internet use; scientific output; bibliometric study; visualization of research fronts.

Trabajo presentado en el IX Seminario de Estudios Cualitativos y Cuantitativos de la Ciencia y la Tecnología "Prof. Gilberto Sotolongo Aguilar", durante el Congreso Internacional de Información INFO'2018, 5 al 9 de marzo de 2018, La Habana, Cuba.

Copyright: ( 2019 CSIC. Este es un artículo de acceso abierto distribuido bajo los términos de la licencia de uso y distribución Creative Commons Reconocimiento 4.0 Internacional (CC BY 4.0). 


\section{INTRODUCCIÓN}

Internet es uno de los hitos tecnológicos de la historia de la humanidad, a partir de la velocidad de su introducción, su expansión global y su penetración masiva en la vida social y profesional de cada ser humano. En su concepto más amplio, Internet abarca las tecnologías de la información y comunicación interconectadas (Web, redes sociales, Internet móvil e Internet de las Cosas, incluyendo la computación en nube, big data y robótica, cada vez más centrales para las tecnologías en red), y ha sido un catalizador de la interacción entre seres humanos (UNESCO, 2017). Internet ha desarrollado nuevas vías para la compartición de información, novedosos servicios para la comunicación, y herramientas efectivas de aprendizaje que han tenido un impacto decisivo tanto en niveles de educación básicos como superiores (Mihajlov y Vejmelka, 2017). Constituye una inmensa esfera intelectual en la que se aprende a explorar libremente todos los saberes, y un foro donde todos los que acceden pueden encontrarse, dialogar, intercambiar y adquirir cultura, conocimientos y valores (Ramonet, 2016).

El ciberespacio creado por Internet superpone a la realidad que vivimos un nuevo universo desarrollado a partir de intercambios digitales de todo tipo, donde cada uno de los espacios públicos conocidos (escuelas, bibliotecas, mercados, medios de comunicación masiva, etc.) son mimetizados en un entorno virtual y están accesibles a través de dispositivos interconectados. La posibilidad de acceder e interactuar fácilmente con estos espacios (desde una cabina pública, una computadora personal o un teléfono inteligente), así como de crear y hacer públicos contenidos individuales, y la calidad de vida que supone el aprovechamiento de las ventajas de su uso, maximiza la percepción pública de Internet como espacio de formación, creatividad y libertad, cuya accesibilidad debe ser asumida como un derecho de todo ser humano.

No obstante, toda nueva tecnología a lo largo de la historia ha tenido sus riesgos y costos asociados, e Internet no es la excepción. Uno de los riesgos que contempla la introducción de esta tecnología está relacionado con su uso inadecuado o excesivo, y la aparición de conductas adictivas que pueden afectar negativamente la salud de un individuo. La adicción a Internet, si bien no ha existido consenso para conceptualmente reconocerla como tal por la comunidad de investigadores en el área de la Psiquiatría (Ellis y otros, 2018; Griffiths, 2018; Ryding y Kaye, 2018), es un fenómeno clínico con potencial para convertirse en un problema de salud mundial, en la medida que aumenta la cantidad de personas con acceso a la red.
Las primeras investigaciones sobre comportamientos adictivos e Internet recogidos en la literatura datan del último lustro del Siglo $X X$, justo cuando la aparición de la World Wide Web convirtió a la red de redes en un fenómeno de masas (Griffiths, 1996; Young, 1996, 1998). Las investigaciones más recientes revelan el carácter complejo de la adicción a Internet, y la necesidad de abordarla desde un enfoque multidisciplinario (Mihajlov y Vejmelka, 2017). En la actualidad, son diversas las revisiones sistemáticas que analizan este padecimiento desde múltiples perspectivas ( $D^{\prime}$ Hondt y Maurage, 2017; De-Sola Gutierrez y otros, 2016; Kuss y Lopez-Fernandez, 2016; Love y otros, 2015; Marchant y otros, 2017; Vondrackova y Gabrhelik, 2016; Zajac y otros, 2017). Sin embargo, a pesar de que existen algunas investigaciones bibliométricas que asumen el uso de Internet en el contexto de las Ciencias de la Salud (Konstantinidis y otros, 2017; Li y otros, 2015; Sweileh y otros, 2017), solo se identificaron dos estudios bibliométricos dedicados a la adicción a Internet, los cuales caracterizaron la producción científica durante los períodos 1996-2005 (Carbonell y otros, 2009) y 2006-2010 (Carbonell y otros, 2016), y constituyen los antecedentes directos del presente trabajo.

Teniendo en cuenta el paulatino e inevitable tránsito del mundo hacia la incorporación de Internet como parte integral de la sociedad (con independencia de las diferencias económicas y socio-políticas entre las naciones), también resulta necesario el conocimiento de los aspectos negativos asociados a su uso. Los sistemas nacionales de salud de cada país, y sus estrategias particulares de abordaje preventivo de las enfermedades que afectan la salud humana, necesitan contar con todos los elementos derivados de la investigación científica que permitan trazar políticas eficientes para minimizar los efectos adversos del uso de Internet. Por tal razón, el presente trabajo pretende caracterizar la investigación dedicada al estudio de los problemas médicos derivados del uso excesivo o inadecuado de esta tecnología, desde una perspectiva bibliométrica. El estudio tiene como objetivos específicos analizar la evolución de la producción científica sobre Internet y sus efectos negativos en la salud humana, así como identificar las principales áreas temáticas que estructuran la investigación sobre la adicción a Internet durante la presente década.

\section{METODOLOGÍA}

En el presente estudio se utilizó PubMed como fuente de datos, por ser la herramienta informativa de primera elección para investigadores y profesionales de las Ciencias Médicas (Lu, 2011). La principal limitación de este portal en el pasado ra- 
dicaba en la indización de los datos de afiliación del primer autor exclusivamente. Sin embargo, esta limitación ha sido superada desde finales de 2013, lo cual ha permitido adjudicar los trabajos a países e instituciones con una mayor precisión.

Como estrategia de búsqueda en PubMed, se utilizó la palabra "Internet" en todos los campos de la base de datos, durante el período comprendido entre los años 2011 y 2017. Se seleccionó el período en aras de poder comparar los resultados obtenidos con los hallazgos de Carbonell y colaboradores en períodos previos (Carbonell y otros, 2009; Carbonell y otros, 2016). Los 43.332 registros recuperados mediante esta estrategia se exportaron a una base de datos ad hoc, utilizando el gestor de referencias bibliográficas EndNote $\mathrm{X} 7$.

Dentro del volumen total de registros, se creó un subgrupo de 29.020 artículos de investigación directamente relacionados con el uso de Internet, a partir de la identificación de la palabra "Internet" en los campos correspondientes al título y palabras clave de los registros. A su vez, se creó un tercer subgrupo de 5.280 artículos dedicados a las investigaciones que tenían Internet como temática principal, a partir de la identificación de la palabra "Internet" en el título de los artículos. Estos tres subgrupos sirvieron como elemento comparativo para valorar el comportamiento de la proporción de la literatura sobre adicción a Internet existente en el mundo durante la presente década. También, se obtuvo un cuarto grupo a partir de la recuperación en la base de datos de los artículos dedicados al estudio de la adicción a Internet.

Para los cuatro grupos obtenidos, se utilizó la siguiente nomenclatura:

1. I-tot (total): artículos recuperados a partir del uso del término "Internet"

2. I-rt (related topic): artículos recuperados a partir del uso del término "Internet" en título y palabras clave

3. I-mt (main topic): artículos recuperados a partir del uso del término "Internet" solo en título

4. IA (Internet addiction): artículos sobre adicción a Internet.

Para la identificación de los artículos sobre adicción a Internet (IA), se tuvo en cuenta la diversidad de criterios relacionados con la asimilación en la nomenclatura oficial del término "adicción", así como la terminología frecuentemente utilizada en la literatura médica para referirse al uso patológico de Internet (Mihajlov y Vejmelka, 2017). A partir de los principales términos identificados, se diseñó una estrategia de búsqueda a implementar en la base de datos ad hoc de 43.332 registros, la cual se expone a continuación:

"Internet addiction OR Internet dependence OR Internet abuse OR Problematic Internet use OR Compulsive Internet use OR Internet gaming disorder OR Internet use disorder OR Internetaddicted patients OR Internet gaming addiction OR Internet game disorder OR Internet addictive disorder OR Excessive use of the Internet OR Excessive use of Internet OR Internet-related addictive behavior OR Pathological internet use OR Online gambling addiction OR Facebook addiction OR Problematic online sex use OR Cyber addiction OR Excessive facebook use OR Addiction to cyberpornography OR Inappropriate use of Internet OR Problematic online game use"

La estrategia persiguió la mayor exhaustividad posible en el proceso de recuperación, y los 1.178 artículos recuperados constituyeron el núcleo de investigaciones que se sometieron al proceso de análisis y visualización mediante técnicas bibliométricas de co-ocurrencia de términos. Particularmente, se emplearon técnicas de co-ocurrencia de palabras en el título.

Se analizó el comportamiento anual de la producción científica sobre adicción a Internet durante la década, y se identificaron las revistas y autores más representativos del dominio. Se utilizó el portal SciMago Journal \& Country Rank (http://www. scimagojr.com) para determinar el cuartil de visibilidad de las revistas líderes del campo, según su índice SJR. Se utilizó el programa Bibexcel para la creación de las matrices de co-ocurrencia de términos, las cuales fueron visualizadas mediante el programa VOSviewer 1.6.5 (Van Eck y Waltman, 2011). El algoritmo VOS, desarrollado para este software por investigadores de la Universidad de Leiden (Holanda), permitió la determinación de grupos en la estructura de las matrices (Van Eck y otros, 2010). La caracterización de los grupos obtenidos facilitó el etiquetado de las principales frentes de investigación relacionadas con la adicción a Internet.

\section{RESULTADOS Y DISCUSIÓN}

La producción científica sobre comportamientos adictivos en línea constituye el $2,7 \%$ de la literatura biomédica mundial asociada a Internet, el 4,1 $\%$ de los artículos de investigación directamente relacionados con el uso de Internet, y el 22,3\% de los trabajos que tienen a Internet como tópico principal. El hecho de que más del $20 \%$ de las investigaciones centradas en Internet como tópico principal se oriente a su uso inapropiado o patológico es indicativo de la importancia que el tema 
reviste para la comunidad científica especializada en las Ciencias Médicas. Tanto la investigación médica focalizada en Internet, como la que estudia los comportamientos adictivos en línea, han crecido durante el período 2011-2017 (Figura 1).

Indiscutiblemente, los resultados obtenidos muestran una evolución significativa con respecto a los estudios previos realizados por Carbonell y colaboradores, que recuperaron apenas 179 artículos en las bases de datos PubMed y Psycinfo durante el período 1996-2005 (Carbonell y otros, 2009), y luego 330 durante el período 2006-2010 (Carbonell y otros, 2016); o sea, se duplicó durante los últimos cinco años estudiados por ellos, la cifra de artículos obtenidos en la década previa.

En ese sentido, a pesar de que el presente trabajo solo tiene en cuenta PubMed, se confirma la proyección del crecimiento de la literatura sobre adicción a Internet, al duplicarse durante el período 2011-2015 (657 artículos) la cifra observada en el lustro anterior; y observarse que el valor de esta cifra es casi similar a lo recuperado solamente durante los años 2016 y 2017 (591 artículos). La tendencia lineal de crecimiento es evidente (Figura 2), y resulta también llamativo que la proporción de trabajos sobre comportamientos adictivos, con respecto al volumen total de investigación médica focalizada en Internet, haya transitado de una décima parte en el año 2011 a un tercio en el año 2017.
Un total de 361 revistas indizadas en PubMed fueron responsables de la publicación de los 1.178 artículos sobre adicción a Internet. De ellas, solo ocho concentraron un tercio de la producción total, y constituyeron el núcleo de publicaciones con mayor nivel de especialización en el tema (Tabla I). Estas ocho revistas más productivas, han tenido un impacto significativo de acuerdo con el índice SJR elaborado por el Grupo SciMago, ocupando el primer cuartil de visibilidad ( $25 \%$ de revistas con mayor impacto) en, al menos, una de las categorías temáticas por las cuales han sido indizadas.

Solo dos de estas revistas, la norteamericana Cyberpsychology \& Behavior (actualmente Cyberpsychology, Behavior, and Social Networking) y la australiana Psychiatry and Clinical Neurosciences coinciden en el grupo de las más productivas identificado por Carbonell y colaboradores en el 2009. De igual forma, la revista norteamericana fue la líder absoluta del lustro 2006-2010 con 73 artículos, y constituye hoy día el principal vehículo de difusión de la investigación sobre adicción a Internet. No obstante, es necesario destacar que durante el período 2011-2017 la diferencia de Cyberpsychology \& Behavior con respecto al resto de las revistas que integran el núcleo más productivo es mucho menor que en los períodos estudiados anteriormente. Este aumento de la productividad en un cada vez mayor número de revistas especializadas, pone en evidencia un área de investigación en pleno desarrollo.

Figura 1. Investigación sobre Internet y Adicción a Internet publicada por la literatura biomédica mundial. PubMed, 2011-2017 (Escala logarítmica)

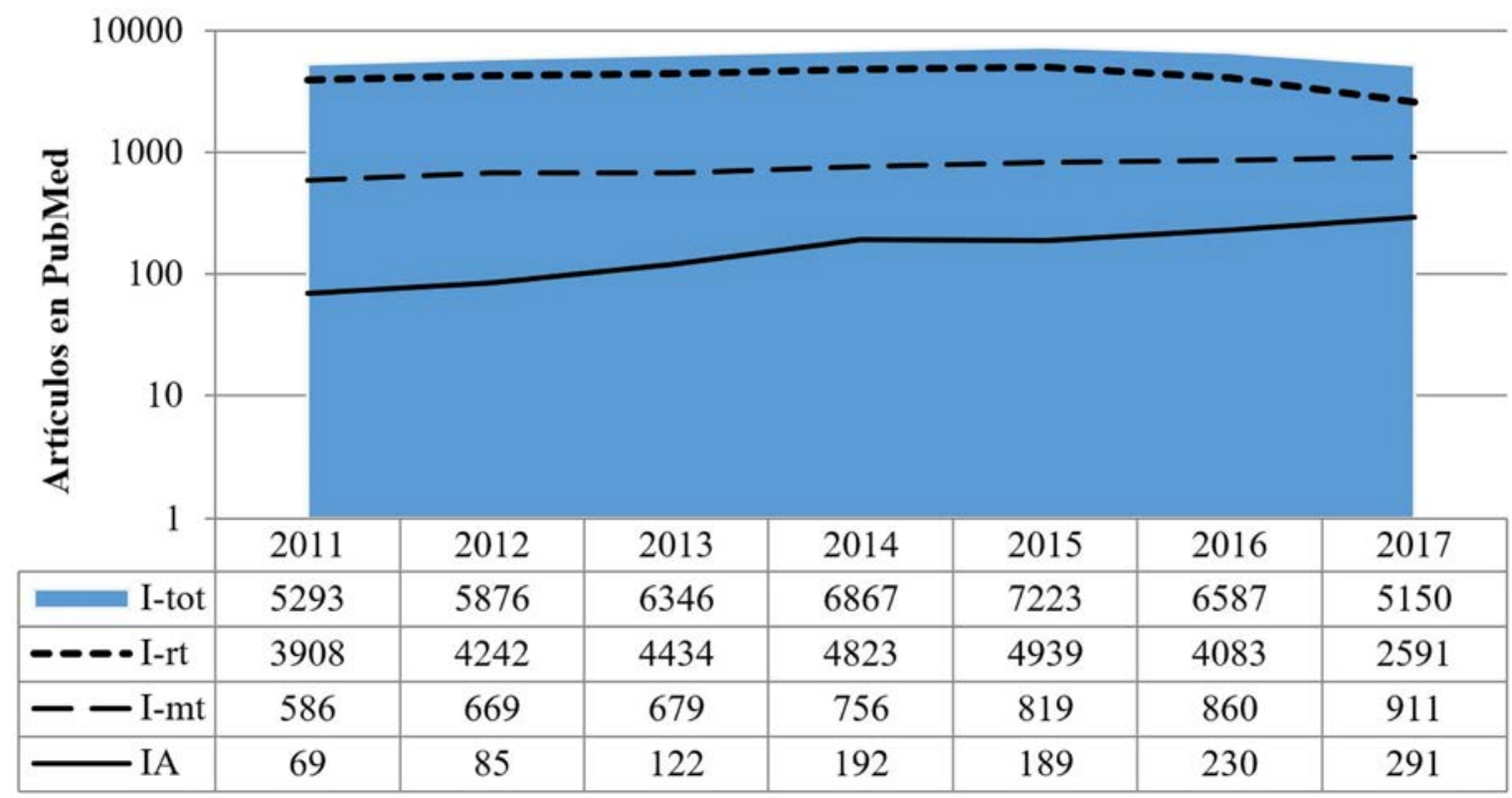


Figura 2. Investigación sobre Adicción a Internet y su evolución en PubMed durante el periodo 2011-2017

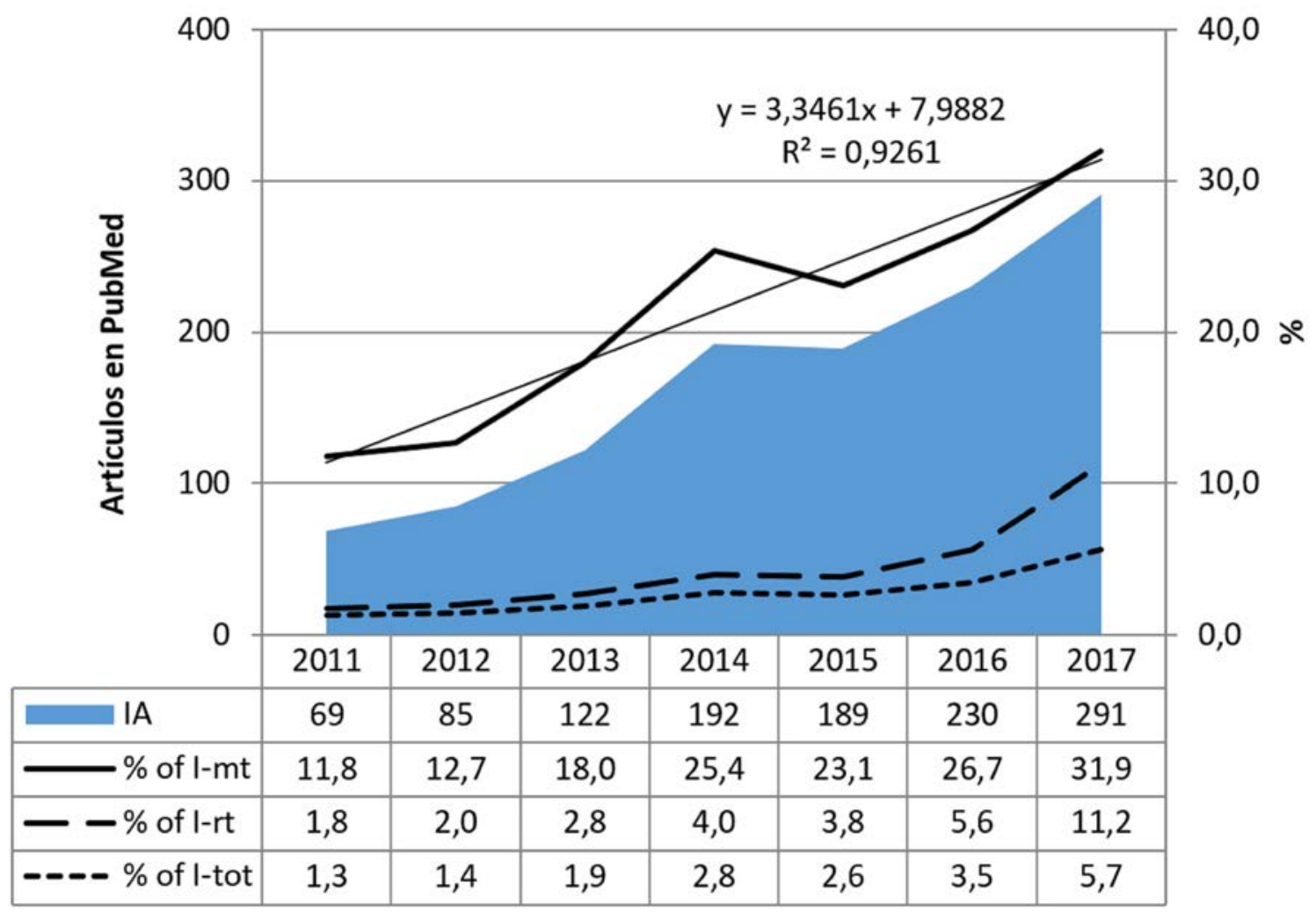

Tabla I. Revistas más productivas sobre adicción a Internet. PubMed, 2011-2017

\begin{tabular}{llcccc}
\hline Revistas más productivas & País & Artículos & \% & SJR & $\begin{array}{c}\text { Cuartil } \\
\text { Scopus }\end{array}$ \\
\hline $\begin{array}{l}\text { Cyberpsychology, Behavior, and Social } \\
\text { Networking }\end{array}$ & Estados Unidos & 100 & 8,5 & 1,298 & Q1 \\
Journal of Behavioral Addictions & Hungría & 81 & 6,9 & 1,101 & Q1 \\
Addictive Behaviors & Inglaterra & 63 & 5,3 & 1,290 & Q1 \\
Psychiatry Research & Irlanda & 45 & 3,8 & 1,215 & Q1 \\
PLoS One & Estados Unidos & 39 & 3,3 & 1.164 & $\mathrm{Q} 1$ \\
Addiction & Inglaterra & 24 & 2,0 & 2,400 & $\mathrm{Q} 1$ \\
Frontiers in Psychology & Suiza & 20 & 1,7 & 1,043 & $\mathrm{Q} 1$ \\
Psychiatry and Clinical Neurosciences & Australia & 17 & 1,4 & 1,231 & $\mathrm{Q} 1$ \\
\hline
\end{tabular}

Por otra parte, si en el primer trabajo de Carbonell y colaboradores, el fenómeno de la adicción a Internet era estudiado por instituciones de tan solo 26 países, encabezados por los Estados Unidos de América (Carbonell y otros, 2009), su siguiente estudio va a mostrar el liderazgo de la República Popular China y la emergencia de otros países asiáticos como Taiwán y Corea del Sur (Carbonell y otros, 2016). En el presente estudio el total de países productores asciende a 56 , liderados por la $\mathrm{Re}$ - pública Popular China (199), Estados Unidos (180), Corea del Sur (129) y Alemania (106), todos con más de 100 artículos publicados. El Reino Unido, Taiwán, Australia, Italia, Turquía y España cierran el grupo de países con más de 30 artículos.

Un total de 3.116 autores contribuyeron a la producción científica sobre adicción a Internet, con un índice de colaboración de 4,86 autores por artículo, muy superior al 2,13 reportado por Carbonell y cola- 
boradores en el 2009. De los más de tres mil autores, solo 24 publicaron 15 o más artículos durante el período, encabezados por uno de los pioneros del estudio de la adicción a Internet: el Dr. Mark D. Griffiths del Departamento de Psicología de la Nothingham Trent University (Reino Unido) (Tabla II). Griffiths publicó 43 artículos durante el período, ha sido uno de los más activos críticos en torno a los aspectos definicionales del campo (Kuss y otros, 2017), y su vasta experiencia en el estudio de la adicción a juegos en línea lo ha llevado a considerar los problemas relacionados con los juegos en línea y los relacionados con el uso de Internet como dos entidades conceptualmente independientes (Kiraly y otros, 2015).

La Figura 3 muestra un conjunto de mapas bibliométricos obtenidos a partir de matrices de coocurrencia de las 382 palabras más repetidas (54 o más repeticiones) en el título de los artículos analizados, en los cuales pueden identificarse ocho grupos temáticos principales. Los grupos se clasificaron de la manera siguiente:

- Cluster 1 (Rojo): Adicción a la ciberpornografía, efectos en la personalidad y las relaciones interpersonales (74 descriptores);
- Cluster 2 (Verde): Alteraciones cognitivas asociadas a la adicción a Internet (70 descriptores);

- Cluster 3 (Azul oscuro): Investigaciones clínicas, instrumentos para la evaluación de la adicción (50 descriptores);

- Cluster 4 (Amarillo): Adicción a videojuegos (comorbilidad en adolescentes) (43 descriptores);

- Cluster 5 (Morado): Adicción a videojuegos (efectos sobre el rendimiento en adultos) (43 descriptores);

- Cluster 6 (Azul acua): Adicción a Internet en jóvenes: relación con alcoholismo, conductas agresivas, ciberbulling y abuso de drogas (37 descriptores);

- Cluster 7 (Azul claro): Estudios nacionales (36 descriptores);

- Cluster 8 (Beige): Sobreuso de teléfonos inteligentes y redes sociales (29 descriptores).

Tabla II. Autores más productivos sobre adicción a Internet. PubMed, 2011-2017

\begin{tabular}{|c|c|c|c|c|}
\hline $\begin{array}{l}\text { Autores más } \\
\text { productivos }\end{array}$ & Institución & País & Artículos & $\%$ \\
\hline Griffiths, M. D. & Nottingham Trent University & Reino Unido & 43 & 3,40 \\
\hline Kim, Don Jun. & The Catholic University of Korea & Corea del Sur & 36 & 3,06 \\
\hline Ko, Chien-Ho & Kaohsiung Medical University & Taiwan & 31 & 2,63 \\
\hline Yen, Cheng-Fang & Kaohsiung Medical University & Taiwan & 30 & 2,55 \\
\hline Choi, Jung-Seok & The Catholic University of Korea & Corea del Sur & 29 & 2,46 \\
\hline Dong, Guangheng & Zhejiang Normal University & China & 27 & 2,29 \\
\hline Zhang, Yifen & Peking University & China & 25 & 2,12 \\
\hline Potenza, Marc N. & Yale University School of Medicine & Estados Unidos & 25 & 1,95 \\
\hline Wolfling, Klaus & Johannes Gutenberg-Universitat Mainz & Alemania & 24 & 1,87 \\
\hline Yen, Ju-Yu & Kaohsiung Medical University & Taiwan & 22 & 1,87 \\
\hline Lee, June-Young & Ajou University School of Medicine & Corea del Sur & 22 & 1,87 \\
\hline King, Daniel L. & The University of Adelaide & Australia & 21 & 1,78 \\
\hline Muller, Kai W. & Johannes Gutenberg-Universitat Mainz & Alemania & 21 & 1,78 \\
\hline Han, Doug Hyun & Chung-Ang University Hospital & Corea del Sur & 21 & 1,78 \\
\hline 24 autores & & & $>14$ & $>1,27$ \\
\hline 122 autores & & & 6 a 14 & 0,51 a 1,19 \\
\hline 709 autores & & & 2 a 5 & 0,17 a 0,42 \\
\hline 2261 autores & & & 1 & 0,08 \\
\hline
\end{tabular}


Figura 3. Red de co-ocurrencia de palabras en el título de los artículos sobre adicción a internet (PubMed, 2011-2017); a) Red de co-ocurrencia de términos; b) Clusters temáticos; c) Mapa de densidad

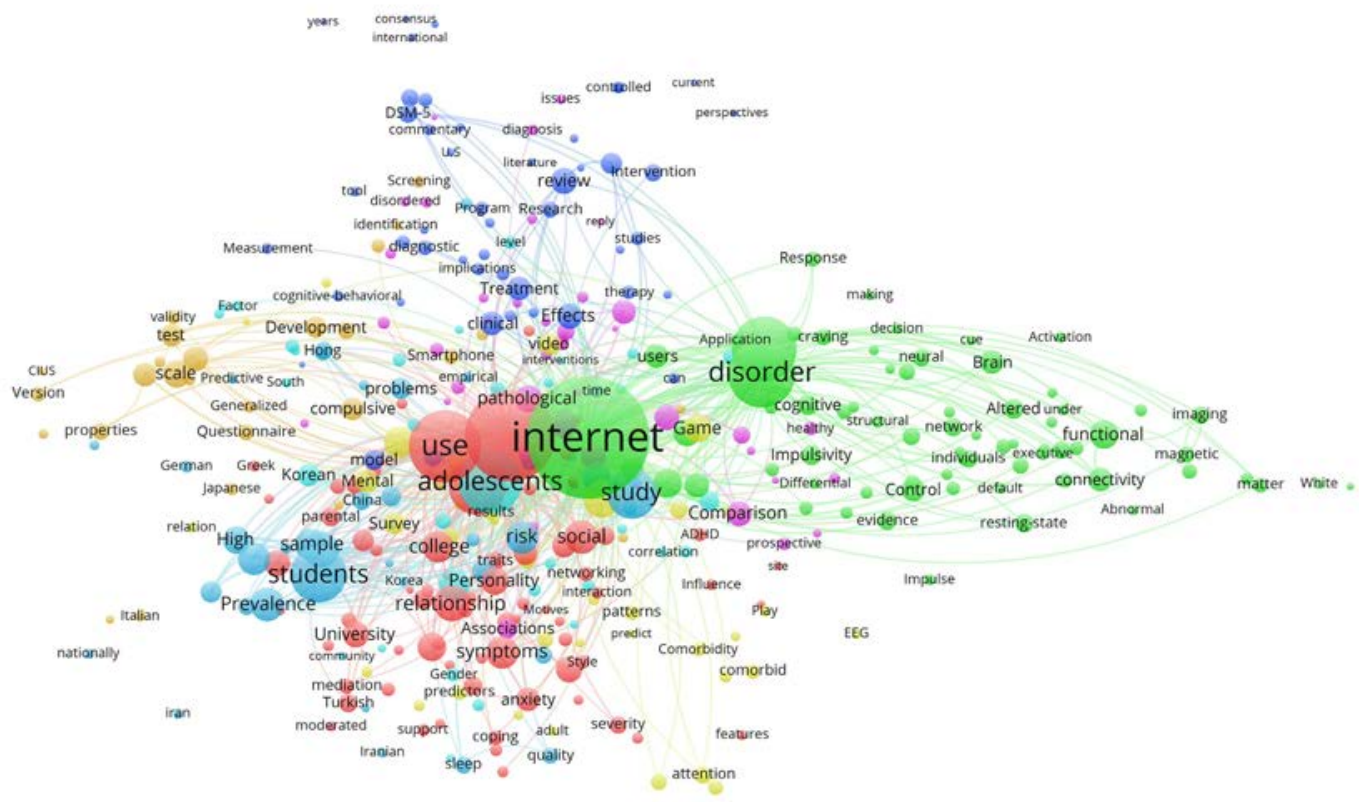

\& vosviewer

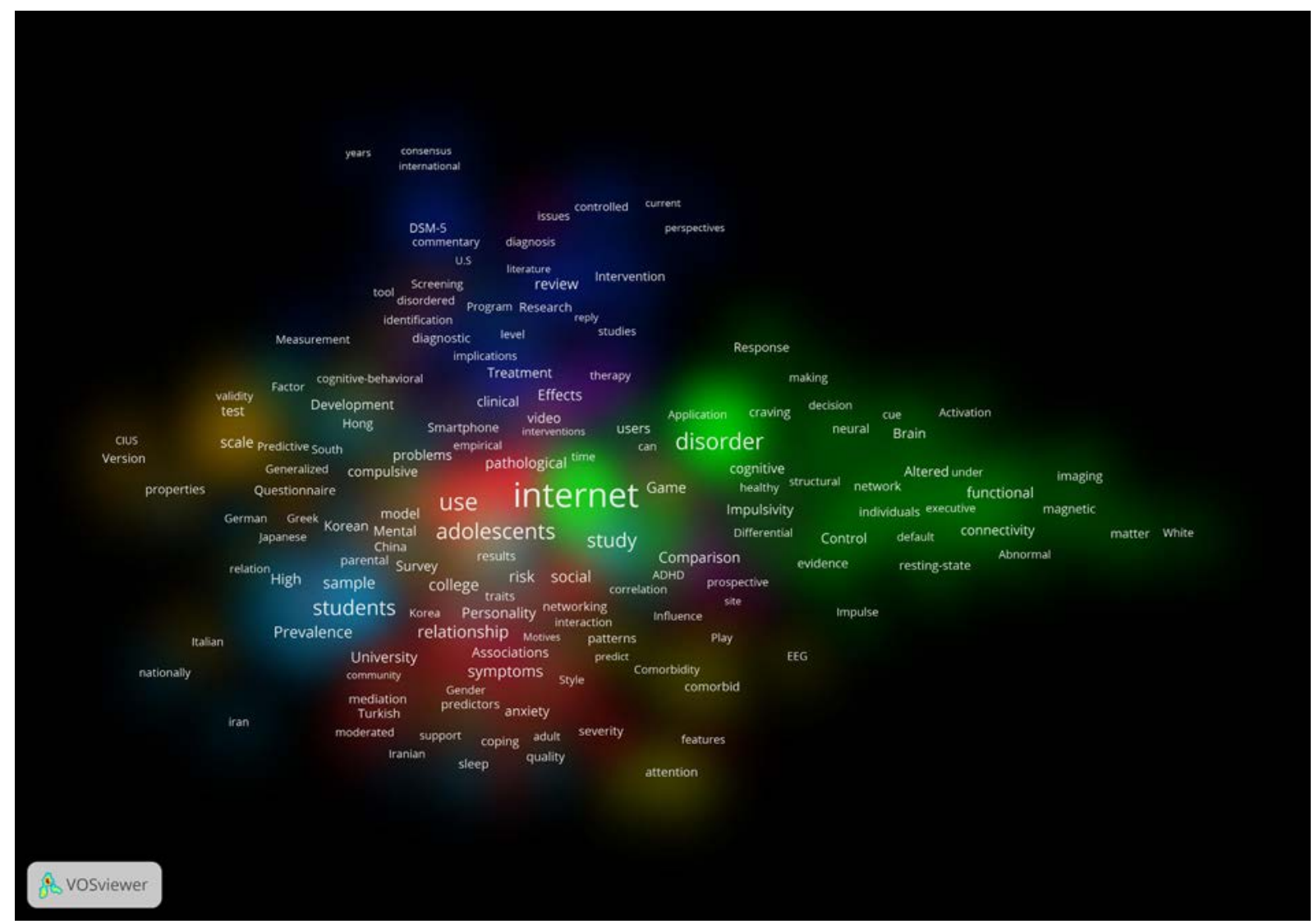




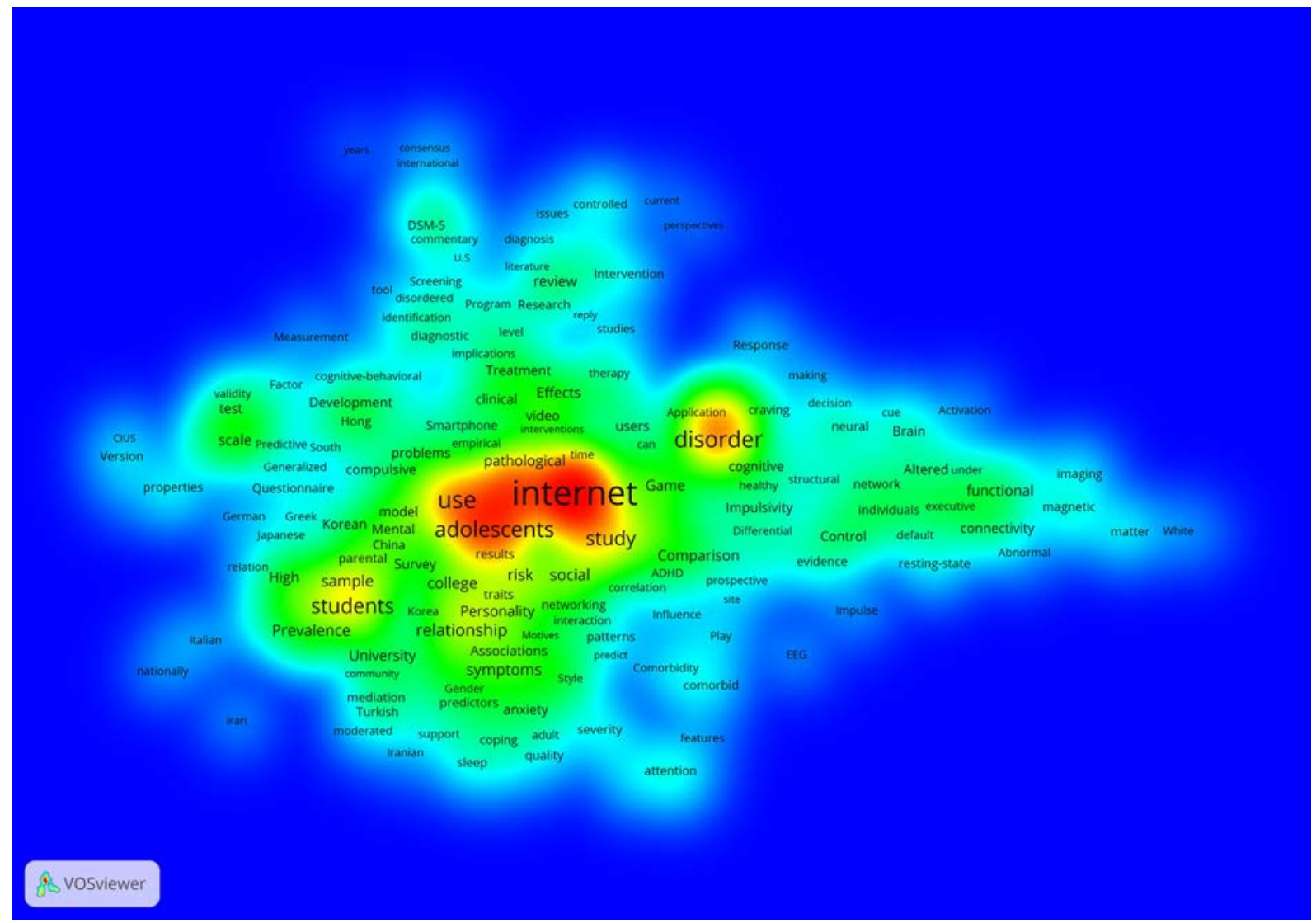

La adicción a los juegos en línea constituye una de las áreas clave de la investigación sobre conductas adictivas en Internet, y es hoy en día el único padecimiento relacionado con Internet oficialmente incluido en el Manual de Diagnóstico y Estadísticas de Desórdenes Mentales (DSM-5), en su quinta revisión, aunque aún como suplemento y no como padecimiento formal (Petry y otros, 2015). Los clúster 4 y 5 obtenidos en el presente estudio, y en cierta medida parte de los grupos 3 y 6 , se enfocan en este problema, que afecta significativamente a niños, adolescentes y jóvenes en edad escolar e incluso laboral (King y otros, 2012), y que incide en gran mayoría en individuos del sexo masculino (Kiraly y otros, 2015).

La adicción a la ciberpornografía, comprendida esencialmente en el clúster 1 , constituye otra temática relevante en el dominio analizado, y es una de las más controvertidas y que mayor cantidad de investigaciones genera a lo largo del período (Allen y otros, 2017; Duffy y otros, 2016). La personalidad y las relaciones interpersonales de individuos con adicción a la pornografía en Internet son aspectos abordados con frecuencia por los estudios, en los que también se observa alta incidencia en individuos del sexo masculino.
Por otra parte, enrolarse excesivamente en relaciones en línea, muy en particular a través de redes sociales ampliamente conocidas como Facebook o Twitter, a partir del uso sistemático y compulsivo de dispositivos móviles, constituye otro de los temas estudiados durante la última década, y representados en el clúster 8. Particularmente, el uso excesivo de redes sociales y teléfonos inteligentes es un fenómeno creciente, que no distingue sexos ni grupos etáreos, aunque puede afectar con más frecuencia a individuos con discapacidades intelectuales (Jenaro y otros, 2017).

Cualquiera de estas tres categorías, de acuerdo a algunos autores, puede considerarse como un caso específico de adicción, lo cual pudiera estar detrás de la no aceptación aún de la adicción a Internet en el Manual de Diagnóstico y Estadísticas de Desórdenes Mentales. Algunos autores discrepan del concepto adicción a Internet como un padecimiento independiente, aspecto observado en algunas investigaciones relacionadas con los clústeres 2 y 7 , enfatizando que Internet es solo el medio a través del cual se manifiestan padecimientos como la ansiedad, la depresión, la soledad social y los problemas de hiperactividad y déficit de atención (ADHD, por sus siglas en inglés) (Mihajlov y Vejmelka, 2017). 
Existen múltiples instrumentos desarrollados para la medición de la adicción a Internet, que involucran diversas escalas, entrevistas y criterios para el diagnóstico. La investigación que utiliza este tipo de instrumentos, claramente identificada en el clúster 3, y la que los aplica a gran escala en entornos nacionales, presente en el clúster 7, no está exenta de críticas, debido a que en muchos casos los cuestionarios se construyen sobre bases teóricas y no tienen validación clínica (Mihajlov y Vejmelka, 2017).

No obstante, los modelos psicopatológicos empleados en las investigaciones han permitido identificar una serie de síntomas (pérdida del sentido del tiempo, irritabilidad, tensión, depresión, ansiedad, fatiga, aislamiento social, tendencia a mentir, etc.), asociados a cualquier otro padecimiento reconocido en el Manual de Diagnóstico y Estadísticas de Desórdenes Mentales. Como criterio diagnóstico, la dedicación de, al menos, 6 horas diarias al uso de Internet (excluyendo los fines académicos, profesionales o de negocios) por un período de 3 meses, de conjunto con la disminución del rendimiento académico o profesional y deterioro de las relaciones interpersonales, son claros síntomas de adicción a Internet (Mihajlov y Vejmelka, 2017).

La búsqueda de alteraciones funcionales en el cerebro mediante neuroimágenes, presente en el clúster 2 , ha permitido a algunos investigadores identificar ciertas características distintivas en el cerebro de adictos al juego en línea con respecto a personas saludables (Dieter y otros, 2017; Wang y otros, 2016). Sin embargo, no existe aún evidencia directa del impacto del uso excesivo de Internet en el cerebro de los niños (Mihajlov y Vejmelka, 2017).

Como puede apreciarse, más allá del reconocimiento o no de la adicción a Internet en el Manual de Diagnóstico y Estadísticas de Desórdenes Mentales, el área de investigación caracterizada desde la perspectiva bibliométrica por Carbonell y cola-

\section{REFERENCIAS}

Allen, A.; Kannis-Dymand, L.; Katsikitis, M. (2017). Problematic internet pornography use: The role of craving, desire thinking, and metacognition. Addictive Behaviors, 70, 65-71. https://doi.org/10.1016/j. addbeh.2017.02.001

Carbonell, X.; Guardiola, E.; Beranuy, M.; Bellés, A. (2009). A bibliometric analysis of the scientific literature on Internet, video games, and cell phone addiction. Journal of the Medical Libraries Association, 97 (2), 102-107. https://doi.org/10.3163/15365050.97.2.006 boradores durante el período 1996-2010 (Carbonell y otros, 2009; Carbonell y otros, 2016), se ha consolidado durante el período 2011-2017, a partir de que los comportamientos adictivos en línea comienzan a constituir un problema de salud emergente, con crecientes tasas de prevalencia en los países que han alcanzado la máxima integración de Internet en el desarrollo de la sociedad (Mihajlov y Vejmelka, 2017).

\section{CONCLUSIONES Y RECOMENDACIONES}

El crecimiento del número de publicaciones científicas que abordan los comportamientos adictivos derivados del uso de Internet pone de manifiesto la existencia de un problema global, que agrupa a su alrededor a un número cada vez mayor de médicos e investigadores. El presente estudio permitió identificar un campo temático en plena expansión, con frentes de investigación bien delimitados, que se difunden en revistas de alta visibilidad. El uso compulsivo de los juegos en línea y la ciberpornografía, y el uso excesivo de redes sociales y teléfonos inteligentes, han sido las principales problemáticas que derivan en adicciones con posibilidad de repercutir negativamente en el comportamiento de los usuarios de la red.

Los problemas intrínsecos del campo, especialmente en los países donde esta tecnología ha logrado los mayores niveles de accesibilidad, ponen de manifiesto la vulnerabilidad de niños, adolescentes y jóvenes a estas adicciones. Por tanto, paralelamente a la gradual y necesaria introducción de Internet en todos los ámbitos de la sociedad, resulta indispensable el desarrollo de políticas públicas que estimulen en la población el aprovechamiento de las múltiples ventajas de su utilización, y minimicen los riesgos de conductas patológicas en estos grupos etarios, las cuales deben movilizar tanto a las familias como a las entidades educativas.
Carbonell, X.; Guardiola, E.; Fuster, H.; Gil, F.; Panova, T. (2016). Trends in Scientific Literature on Addiction to the Internet, Video Games, and Cell Phones from 2006 to 2010. International Journal of Preventive Medicine, 7 (1), 63. https://doi.org/10.4103/20087802.179511

D'Hondt, F.; Maurage, P. (2017). Electrophysiological studies in Internet addiction: A review within the dualprocess framework. Addictive Behaviors, 64, 321-327. https://doi.org/10.1016/j.addbeh.2015.10.012 
De-Sola Gutierrez, J.; Rodriguez de Fonseca, F.; Rubio, G. (2016). Cell-Phone Addiction: A Review. Frontiers in Psychiatry, 7, 175. https://doi.org/10.3389/fpsyt. 2016.00175

Dieter, J.; Hoffmann, S. ; Mier, D. ; Reinhard, I. ; Beutel, M. ; Vollstadt-Klein, S.; Kiefer, F.; Mann, K.; Leménager, T. (2017). The role of emotional inhibitory control in specific internet addiction - an fMRI study. Behavioural Brain Research, 324, 1-14. https://doi.org/10.1016/j. bbr.2017.01.046

Duffy, A.; Dawson, D. L.; das Nair, R. (2016). Pornography Addiction in Adults: A Systematic Review of Definitions and Reported Impact. The Journal of Sexual Medicine, 13 (5), 760-777. https://doi.org/10.1016/j. jsxm.2016.03.002

Ellis, D. A. ; Kaye, L. K.; Wilcockson, T. D. W. ; Ryding, F. C. (2018). Digital Traces of Behaviour Within Addiction: Response to Griffiths (2017). International Journal of Mental Health and Addiction, 16, 240-245. https://doi. org/10.1007/s11469-017-9855-7

Griffiths, M. D. (1996). Internet "addiction": an issue for clinical psychology? Clinical Psychology Forum, 76, 14-19.

Griffiths, M. D. (2018). Conceptual Issues Concerning Internet Addiction and Internet Gaming Disorder: Further Critique on Ryding and Kaye (2017). International Journal of Mental Health and Addiction, 16, 233-239. https://doi.org/10.1007/s11469-017-9818-z

Jenaro, C.; Flores, N.; Cruz, M.; Perez, M. C.; Vega, V.; Torres, V. A. (2017). Internet and cell phone usage patterns among young adults with intellectual disabilities. Journal of Applied Research in Intellectual Disabilities, 31 (2), 259-272. https://doi.org/10.1111/jar.12388

King, D. L.; Delfabbro, P. H.; Griffiths, M. D.; Gradisar, M. (2012). Cognitive-behavioral approaches to outpatient treatment of internet addiction in children and adolescents. Journal of Clinical Psychology, 68(11), 11851195. https://doi.org/10.1002/jclp. 21918

Kiraly, O.; Urban, R.; Griffiths, M. D.; Agoston, C.; Nagygyorgy, K.; Kokonyei, G.; Demetrovics, Z. (2015). The mediating effect of gaming motivation between psychiatric symptoms and problematic online gaming: an online survey. Journal of Medical Internet Research, 17 (4), e88. https://doi.org/10.2196/jmir.3515

Konstantinidis, S. T.; Billis, A.; Wharrad, H.; Bamidis, P. D. (2017). Internet of Things in Health Trends Through Bibliometrics and Text Mining. Studies in Health Technology and Informatics, 235, 73-77.

Kuss, D. J.; Griffiths, M. D.; Pontes, H. M. (2017). Chaos and confusion in DSM-5 diagnosis of Internet Gaming Disorder: Issues, concerns, and recommendations for clarity in the field. Journal of Behavioral Addictions, 6 (2), 103-109. https://doi. org/10.1556/2006.5.2016.062

Kuss, D. J.; ' Lopez-Fernandez, O. (2016). Internet addiction and problematic Internet use: A systematic review of clinical research. World Journal of Psychiatry, 6 (1), 143-176. https://doi.org/10.5498/wjp.v6.i1.143
Li, F.; Li, M.; Guan, P.; Ma, S.; Cui, L. (2015). Mapping publication trends and identifying hot spots of research on Internet health information seeking behavior: a quantitative and co-word biclustering analysis. Journal of Medical Internet Research, 17 (3), e81. https://doi. org/10.2196/jmir.3326

Love, T.; Laier, C.; Brand, M.; Hatch, L. ; Hajela, R. (2015). Neuroscience of Internet Pornography Addiction: A Review and Update. Behavioral Sciences, 5 (3), 388-433. https://doi.org/10.3390/bs5030388

Lu, Z. (2011). PubMed and beyond: a survey of web tools for searching biomedical literature. Database, 2011, baq036. https://doi.org/10.1093/database/baq036

Marchant, A.; Hawton, K.; Stewart, A.; Montgomery, P.; Singaravelu, V.; Lloyd, K.; Purdy, N.; Daine, K.; John, A. (2017). $A$ systematic review of the relationship between internet use, self-harm and suicidal behaviour in young people: The good, the bad and the unknown. PLoS One, 12 (8), e0181722. https://doi.org/10.1371/journal.pone.0181722

Mihajlov, M.; Vejmelka, L. (2017). Internet addiction: a review of the first twenty years. Psychiatria Danubina, 29 (3), 260-272.

Petry, N. M.; Rehbein, F.; Ko, C. H.; O'Brien, C. P. (2015). Internet Gaming Disorder in the DSM-5. Current Psychiatry Reports, 17 (9), 72. https://doi. org/10.1007/s11920-015-0610-0

Ramonet, I. (2016). El imperio de la vigilancia. La Habana: Editorial José Martí.

Ryding, F. C.; Kaye, L. K. (2018). Internet Addiction: a Conceptual Minefield. International Journal of Mental Health and Addiction, 16, 225-232. https://doi. org/10.1007/s11469-017-9811-6

Sweileh, W. M.; Al-Jabi, S. W.; AbuTaha, A. S.; Zyoud, S. H.; Anayah, F. M. A.; Sawalha, A. F. (2017). Bibliometric analysis of worldwide scientific literature in mobile - health: 2006-2016. BMC Medical Informatics and Decision Making, 17 (1), 72. https://doi.org/10.1186/ s12911-017-0476-7

UNESCO. (2017). Las piedras angulares para la promoción de sociedades del conocimiento inclusivas: Acceso a la información y al conocimiento, libertad de expresión, privacidad y ética en la Internet global. París: Organización de las Naciones Unidas para la Educación, la Ciencia y la Cultura.

Van Eck, N. J.; Waltman, L. (2011). Text mining and visualization using VOSviewer. ISSI Newsletter, 7 (3), 50-54.

Van Eck, N. J.; Waltman, L.; Dekker, R.; Van den Berg, J. (2010). A comparison of two techniques for bibliometric mapping: Multidimensional scaling and VOS. Journal of the American Society for Information Science and Technology, 61 (12), 2405-2416. https://doi. org/10.1002/asi.21421

Vondrackova, P.; Gabrhelik, R. (2016). Prevention of Internet addiction: A systematic review. Journal of Behavioral Addictions, 5 (4), 568-579. https://doi. org/10.1556/2006.5.2016.085 
Wang, L.; Wu, L.; Lin, X.; Zhang, Y.; Zhou, H.; Du, X.; Dong, G. (2016). Altered brain functional networks in people with Internet gaming disorder: Evidence from resting-state fMRI. Psychiatry Research, 254, 156-163. https://doi.org/10.1016/j.pscychresns.2016.07.001

Young, K. (1996). Psychology of computer use: XL. Addictive use of the Internet: a case that breaks the stereotype. Psychology Report, 79, 899-902.
Young, K. (1998). Caught in the Net: How to recognize the signs of Internet addiction and a winning strategy for recovery. New York: Wiley.

Zajac, K.; Ginley, M. K.; Chang, R.; Petry, N. M. (2017). Treatments for Internet Gaming Disorder and Internet Addiction: A Systematic Review. Psychology of Addictive Behaviors, 31 (8):979-994. https://doi. org/10.1037/adb0000315 